intercourse (AI) in the last 90 days. Of these women, only 113 $(58.2 \%)$ also ordered and returned rectal kits for testing. An additional 95 kits were ordered and returned by women who did not report recent AI (82), did not return a vaginal swab (5), or did not answer the AI question on the vaginal questionnaire (8). From a total of 406 rectal kits ordered by women overall, 208 (51.2\%) were returned; three had no consent form; thus, 205 were tested. Of those tested, $26(12.7 \%)$ were positive for chlamydia, $5(2.4 \%)$ were positive for gonorrhoea, and 13 (6.3\%) were positive for trichomoniasis. Two of these samples were positive for both Chlamydia and gonorrhoea, two for both chlamydia and trichomonas, and one for all three STIs. The total number of women testing positive for any rectal STI was 38 (18.5\%), and 35 of these women also received and returned vaginal swabs, 34 of which were tested. Of those tested, 24 $(70.5 \%)$ were positive for at least one of the three STIs vaginally, indicating that women who tested positive for rectal STIs were at very high risk for vaginal STIs. Of the 38 women with rectal STIs, the median age was $22 \mathrm{yr}$. and the median age of first rectal sex was 20 yr. Questionnaires demonstrated 93.6\% were single, $62.5 \%$ were Black, and $21.9 \%$ were White. Of the women with rectal STIs, $67.7 \%$ reported no symptoms, $12.0 \%$ reported no rectal partners in previous yr, $56.0 \%$ reported one partner, and 32\% reported $2-4$ partners. Only $16.0 \%$ reported having a new rectal partner in the last 3 months. Half (50.0\%) reported never using condoms, $15.4 \%$ reported they always used condoms, $15.4 \%$ reported using condoms most of the time, and $19.2 \%$ reported using condoms some of the time.

Conclusions Public health officials should be aware that AI and rectal STIs are not uncommon among sexually active women. Future STI screening programs should consider rectal infections.

\section{1-S07.03 PREVALENCE AND CORRELATES OF RECTAL CHLAMYDIA AND GONORRHOEA AMONG FEMALE STD CLINIC CLIENTS}

\section{doi:10.1136/sextrans-2011-050109.39}

${ }^{1} \mathrm{M}$ Javanbakht, ${ }^{2} \mathrm{~S}$ Guerry, ${ }^{2} \mathrm{~A}$ Stirland, ${ }^{3} \mathrm{P}$ Gorbach, ${ }^{2} \mathrm{P}$ Kerndt. ${ }^{1}$ University of California Los Angeles, Los Angeles, USA; ${ }^{2}$ Los Angeles County Department of Public Health, USA; ${ }^{3}$ University of Calinfornia, Los Angelesm, USA

Background The prevalence and correlates of rectal sexually transmitted infections (STI) are well described among men who report receptive anal intercourse (AI). However, very little is known about the epidemiology of rectal STIs among women.

Methods We conducted a cross-sectional study of women attending twelve public STD clinics in Los Angeles County, California. Women were eligible for inclusion in this study if they reported $\mathrm{AI}$ in the previous 90 days, were tested for rectal chlamydia (CT) and gonorrhoea (GC), and were seen between January 2008 and December 2009. Data collected included demographics, types of sexual contact, substance use, other risk behaviours, and STI results. Results Among the 716 females included in this analysis, the median age was 28 years (range 14-60), 40\% were Hispanic, 37\% African American, and 15\% White. The prevalence of CT and GC by anatomic site was: $11.8 \%(n=83)$ urogenital CT, 14.2\% $(n=100)$ rectal CT, $2.7 \%(n=19)$ urogenital GC, and $3.0 \%(n=21)$ rectal GC. Among positives, $27 \%$ of CT cases and $27 \%$ of GC cases had rectal only infections. Among women $=25$ years the prevalence of rectal infections was higher among those incarcerated in the past year $(39.4 \%$ vs $22.1 \%$; $p$ value $=0.03)$ and those who reported sex with an injection drug user $(50.0 \%$ vs $23.3 \%$; $p$ value $=0.05)$. Among women $>25$ years the prevalence of rectal infections was higher among those with longer term sex partners (ie, $>90$ days; $12.6 \%$ vs $5.9 \%$; value $=0.03)$, and those who reported substance use $(14.8 \%$ vs $8.3 \%$; $p$ value $=0.04)$. While the number of women with a rectal-only infections (ie, no urogenital infection; $n=33$ ) was small, similar trends were observed. After controlling for age and race/ethnicity, among women $\geq 25$ years those incarcerated in the previous year were more likely to have a rectal infection (adjusted OR [AOR]= 2.55 ; $95 \%$ CI $1.14 \%$ to $5.71 \%$ ). Among women $>25$ years, after controlling for race/ethnicity and age, those who reported substance use were more likely to have a rectal infection ( $\mathrm{AOR}=2.28 ; 95 \% \mathrm{CI}$ $1.14 \%$ to $4.55 \%$ ) while those reporting new sex partners were less likely to have a rectal infection $(\mathrm{AOR}=0.38 ; 95 \% \mathrm{CI} 0.16 \%$ to $0.88 \%)$.

Conclusion These findings highlight that the prevalence of rectal Chlamydia and gonorrhoea among women is similar to that of urogenital infections and a relatively large number of infections would be missed in the absence of rectal testing. Factors associated with rectal infections may differ by age.

\section{1-S07.04 MANY MISSED STI WHEN ONLY TESTING UROGENITALLY WITHOUT SYSTEMATIC ANORECTAL AND OROPHARYNGEAL SCREENING IN SWINGERS AND MSM}

doi:10.1136/sextrans-2011-050109.40

${ }^{1,2} \mathrm{C}$ Hoebe, ${ }^{3} \mathrm{G}$ van Liere, ${ }^{4} \mathrm{~A} \mathrm{M}$ Niekamp, ${ }^{1} \mathrm{~N}$ Dukers-Muijrers. ${ }^{1}$ Department of Infectious Diseases, South Limburg Public Health Service, Maastricht University Medical Centre, School for Public Health and Primary Care Geleen, Netherlands, ${ }^{2}$ Department of Medical Microbiology, Maastricht University Medical Centre, School for Public Health and Primary Care Geleen, Netherlands; ${ }^{3}$ Department of Infectious Diseases, South Limburg Public Health Service Geleen, Netherlands; ${ }^{4}$ Centre for Sexual Health, Department of Infectious Diseases, Public Health Service South Limburg, Netherlands

Background Currently, risk groups are tested only urogenitally by most healthcare providers like general practitioners and STI clinics. Only on indicationmostly guided by symptomsanorectal and/or oropharyngeal tests are performed. Patient identification and thereby adequate treatment and interruption of the chain of transmission can be hampered when patients are positive at another anatomic site than sampled for testing. It is unknown how large this potentially missed STI burden in healthcare is. In this study we assessed the burden of undetected STI in high risk heterosexuals (swingers) and men who have sex with men (MSM), based on systematic testing at three anatomical sites.

Methods All MSM and high risk heterosexuals that is, swingers, who as a couple have sex with other couples, were systematically screened for urogenital, anorectal and oropharyngeal STI at our STI Centre in 2010. This comprised 762 swinger consultations and 597 (non swinging) MSM consultations. One third of the male swingers reported to have sex with men. Prevalences of Chlamydia trachomatis (CT) and Neisseria gonorrhoea (NG), and STI (CT and/or NG) were calculated on multiple anatomical sites as well as the proportion of anorectal and oropharyngeal diagnosis that would have been missed if screened urogenital only.

Results Prevalences were $7 \%$ and $10 \%$ for CT, and $3 \%$ and $5 \%$ for NG in swingers and MSM, respectively (Abstract O1-S07.04 table 1). Of all anorectal CT in MSM $86 \%(n=42)$ was diagnosed without an urogenital CT, for NG this was $65 \%(n=11)$. Seventy-five per cent $(n=6)$ of all oropharyngeal CT and $69 \%(n=11)$ of all oropharyngeal NG diagnoses would have been missed if MSM had been tested urogenital only. Prevalence of anorectal CT was higher than that of urogenital CT in female swingers. The proportions of missed anorectal diagnosis for CT were 50\% (n=4) and 24\% $(n=5)$ for male and female swingers, respectively. No anorectal CT diagnosis was missed in male swingers, but in female swingers this was $67 \%$ $(n=2)$. All oropharyngeal CT $(n=6)$ and nearly all NG $(91.7 \%$, $\mathrm{n}=11$ ) infections in swingers were diagnosed without a urogenital infection. 